\title{
Oral exclusive enteral nutrition for induction of clinical remission, mucosal healing, and improvement of nutritional status and growth velocity in children with active Crohn's disease - a prospective multicentre trial
}

\author{
Małgorzata Matuszczyk ${ }^{1}$, Monika Meglicka ${ }^{1}$, Piotr Landowski², Elżbieta Czkwianianc ${ }^{3}$, Beata Sordyl ${ }^{3}$, \\ Edyta Szymańska ${ }^{1}$, Jarosław Kierkuś ${ }^{1}$ \\ ${ }^{1}$ Department of Gastroenterology, Hepatology, Feeding Disorders and Paediatrics, The Children's Memorial Health Institute, \\ Warsaw, Poland \\ ${ }^{2}$ Department of Gastroenterology, Allergology and Paediatric Nutrition, Medical University of Gdansk, Gdansk, Poland \\ ${ }^{3}$ Department of Gastroenterology, Allergology and Paediatrics, Polish Mother's Memorial Hospital - Research Institute, Lodz, Poland
}

Key words: paediatric Crohn's disease, nutritional therapy, remission, nutritional status, exclusive enteral nutrition.

Address for correspondence: Malgorzata Matuszczyk MSc, Department of Gastroenterology, Hepatology, Feeding Disorders and Paediatrics, The Children's Memorial Health Institute, Warsaw, Poland, e-mail: m.matuszczyk@ipczd.pl

\begin{abstract}
Introduction: Undernutrition and growth failure are common problems in paediatric patients with active Crohn's disease (CD). Aim: The aim of exclusive enteral nutrition (EEN) commencement is not only to induce clinical remission and promote mucosal healing but also to initiate weight and growth gain, especially in patients with poor nutritional status. We assessed the effectiveness of treatment with EEN and its impact on nutritional status in children with active CD.

Material and methods: Twenty children (male/female: 14/6) in median age of 14 years with active CD had EEN with polymeric industrial diet (Modulen IBD) applied for 6 weeks. The daily caloric intake was established according to the age and nutritional status. In patients with undernutrition, it was increased to $120-150 \%$ relative to recommendations for the healthy peers. The Paediatric CD activity index (PCDAl) - a marker of clinical remission, faecal calprotectin (FCP) - a marker of mucosal healing $(\mathrm{MH})$, and nutritional status were assessed at baseline and 4 weeks following the end of the therapy (week 10).

Results: In the studied group the mean decrease in PCDAl score was statistically significant (from $25.6 \pm 12$ to $5.4 \pm 10, p<$ 0.05). Full remission (defined as PCDAI $<10$ ) was achieved in $65 \%$ of patients, and clinical response in another $30 \%$ of them. Only $5 \%$ of children did not respond to the treatment. Mean decline in FCP level was statistically significant as well (from 3380 \pm 7746 to $1046.6 \pm 1219, p<0.05$ ). All patients, apart from one who was fed with a nasogastric tube, accepted oral intake of industrial formula. EEN was generally well tolerated. Initially, in $20 \%$ of patients the symptoms of intolerance to the industrial diet were observed, but they receded within the first days of the therapy. The recommended daily intake of the formula was achieved in $95 \%$ of children. Only one child was unable to intake the prescribed amount of the diet due to intolerance. At baseline, undernutrition was observed in $30 \%$ of patients, which was established by a body mass index (BMI) score below the third percentile according to the recommended charts for the Polish paediatric population. In all patients, improvement in BMI status was reported at the end of the treatment. The mean increase in BMI score was 0.91, and it was greater in the malnourished group compared to patients with normal nutritional status (1.19 vs. 0.62). After the treatment two-thirds of children with malnutrition achieved a BMI score within the normal range. In $25 \%$ of patients, growth deficit was observed (defined as growth below the third percentile according to the Polish charts) before the EEN introduction. An increase in body height was obtained generally in $55 \%$ of children and in $80 \%$ of those with initial growth failure. The mean increase in growth was $1 \mathrm{~cm}$, and it was greater in the group with initial growth deficit relative to patients with baseline normal height $(1.5 \mathrm{~cm}$ vs. $0.8 \mathrm{~cm}$, respectively).

Conclusions: A 6-week course of oral EEN was an effective and well-tolerated method of treatment in children with active CD. Nutritional therapy not only induced full clinical remission and led to decline in FCP level (as a marker of $\mathrm{MH}$ ) in the majority of patients, but also contributed to the improvement in their nutritional status and growth velocity. These are very important observations because proper development is crucial for paediatric CD patients.
\end{abstract}




\section{Introduction}

Crohn's disease (CD) is a chronic, incurable inflammatory disease of the gastrointestinal tract, usually diagnosed during childhood or adolescence [1]. Growth failure and malnutrition are typical complications of inflammatory bowel disease (IBD) in children. Its aetiology is multifactorial, but there are two main factors contributing to that state: chronic intestinal inflammation leading to poor absorption, which finally causes malnutrition, and the direct effect of proinflammatory cytokines secreted by inflamed intestine [2, 3].

The treatment strategies for CD in children have evolved markedly in recent years. Two meta-analyses have shown that exclusive enteral nutrition has comparable effectiveness to traditional corticosteroid therapy in the treatment of children with active $C D[4,5]$. It should also be emphasized that steroid therapy has many side effects, including a negative impact on bone growth and mineralization processes, which is particularly important in children. Exclusive enteral nutrition (EEN) not only contributes to growth gain but also improves the skeletal system condition [6]. What is more, the latest meta-analysis (2017) showed not only the comparable effectiveness of EEN and corticosteroids in the induction of clinical remission in children with active $C D$, but also a higher efficiency of EEN in the induction of mucosal healing [7]. These findings were confirmed in 2019 by Pigneur et al.; in their study clinical remission was achieved in $100 \%$ patients on EEN and in $83 \%$ patients on steroids, but the mucosal healing rate was significantly higher in the EEN group (89\%) compared with the steroid group (17\%) [8]. It is known that obtaining mucosal healing in patients with CD is associated with better outcomes in long-term observation [9-11].

Similar effectiveness in induction of remission, no side effects, positive effect on child's development and better efficiency in induction of mucosal healing are factors in favour of using EEN instead of corticosteroid therapy as a first-line treatment for young patients with active $C D$. On the other hand, it is known that in clinical practice EEN is not a convenient and easy therapeutic method to commence. The necessity for the patient's high motivation, especially when the nasogastric tube placement is needed, is a common reason for renouncement this kind of treatment in children and adolescents.

There are several potential mechanisms of the impact of EEN on the inflamed intestinal mucosa, including the avoidance of enteral antigens resulting in improvement in intestinal permeability or beneficial changes to the gut microbiome [12-14]. Also, the industrial diet directly affects intestinal mucosa through reduction of secretion of pro-inflammatory cytokines [15]. Numerous studies have analysed the impact of modifications in industrial diet composition, including changes in the type of fat, protein, and bioactive peptide content/addition, but there is still no consensus on the most appropriate type of formula for CD patients $[5,16]$. According to current guidelines, generally the standard polymeric formula is recommended [17].

However, it seems that the addition of bioactive peptides to the industrial diet may give additional benefits in the treatment of CD due to their anti-inflammatory effect $[13,18]$. Bioactive peptides are specific growth factors. Transforming growth factor- $\beta$ (TGF- $\beta$ ) comprises multifunctional regulatory peptides produced by various cell types. It is known that TGF- $\beta$ affects the immune system regulation, as well as cell growth and differentiation both through autocrine and paracrine pathways. These cytokines control the processes of cell differentiation, proliferation, and activation (including lymphocytes, macrophages, and dendritic cells). Therefore, TGF- $\beta$ plays a key role in anti-inflammatory processes and in prevention of autoimmune diseases [19]. Due to the therapeutic and anti-inflammatory properties of TGF- $\beta$, it seems that its addition to the industrial diet may be effective in CD treatment $[20,21]$.

Currently, only a few paediatric cohort studies analysing the effect of enteral nutrition based on formulas with TGF- $\beta$ (MODULEN IBD) are available [8, 15, 22-24]. In all studies, patients receiving MODULEN IBD entered clinical remission. However, differences in duration of the therapy, method of industrial diet administration (oral/through nasogastric tube), and its daily amount (exclusive/partial enteral nutrition) limit the ability to summarize the results. Moreover, there are only 3 studies on EEN with an industrial diet provided for several weeks, which according to the current guidelines is considered the most effective method of treatment. The same 3 studies are also the only ones that assessed the impact of EEN on mucosal healing [8, 22, 24].

\section{Aim}

Therefore, there is still a need for properly designed studies determining whether total enteral nutrition based on the industrial diet with TGF- $\beta$ is effective in the induction of clinical remission and mucosal healing in children with active CD.

\section{Material and methods}

Twenty patients with active CD (based on clinical symptoms, The Paediatric CD activity index (PCDAI) score, faecal calprotectin (FCP) level, and endoscopic examination) in the age group 4-18 years were included in the study.

The qualification for nutritional treatment was made after the full endoscopic examination (both gastro- and 
colonoscopy were performed), and the SES-CD scale was used to assess macroscopic disease activity.

Exclusive enteral nutrition was provided for 6 weeks to all children. Patients received "Modulen IBD" orally or through a nasogastric tube in cases of intolerance to oral supply. In addition to the industrial diet, drinking of water was allowed.

Daily caloric intake was established based on patient's age and nutritional status. In malnourished patients, daily caloric intake was increased to $120-150 \%$ relative to recommendations for healthy peers (based on body weight, height, and body mass index (BMI) for age and sex).

Clinical remission was defined as $P C D A I<10$, and mucosal healing $(\mathrm{MH})$ was established using FCP level ( $n<200 \mathrm{mg} / \mathrm{kg}$ ).

Nutritional state, PCDAl score, and FCP were assessed at baseline and 4 weeks after the therapy (week 10).

\section{Results}

Among 20 children included in the study, there was a predominance of boys: male/female $-14 / 6$. The range of patients' age was $8.6-17.1$ years, and medi-

Table I. Patients' baseline characteristics $(n=20)$

\begin{tabular}{|c|c|}
\hline Baseline & Results \\
\hline Males & $14(70 \%)$ \\
\hline Age at onset [years] & $12.65 \pm 2.38$ \\
\hline Age range at onset [years] & $8.6-17.1$ \\
\hline Age at baseline [years] & $14.16 \pm 1.96$ \\
\hline Age range at baseline [years] & $9.9-17.5$ \\
\hline New-onset disease & $8(40 \%)$ \\
\hline $\begin{array}{l}\text { Disease duration in recurrence group [years] } \\
(n=12)\end{array}$ & $2.3 \pm 1.8$ \\
\hline $\begin{array}{l}\text { Time from onset/recurrence the symptoms to } \\
\text { baseline [days] }\end{array}$ & $69 \pm 66$ \\
\hline \multicolumn{2}{|l|}{ Location of disease (Paris): } \\
\hline L1-ileal/ileocecal & $16(80 \%)$ \\
\hline L3-ileocolonic & $2(10 \%)$ \\
\hline $\mathrm{L} 4 \mathrm{a}+\mathrm{L} 1$ & $2(10 \%)$ \\
\hline \multicolumn{2}{|l|}{ Severity: } \\
\hline \multicolumn{2}{|l|}{ Baseline PCDAI: } \\
\hline Mild (7.5-27.5) & $13(65 \%)$ \\
\hline Moderate (30-37.5) & $4(20 \%)$ \\
\hline Severe $(>40)$ & $3(15 \%)$ \\
\hline Baseline foetal calprotectin > $200[\mathrm{mg} / \mathrm{kg}]$ & $18(90 \%)$ \\
\hline $\begin{array}{l}\text { Nutritional status }(\mathrm{BMI}) \\
\text { Undernutrition }=\mathrm{BMI}<3 \mathrm{pp}\end{array}$ & $\begin{array}{l}30 \% \text { of } \\
\text { patients }\end{array}$ \\
\hline
\end{tabular}

pp-percentile. an age was 14 years. At baseline the majority of patients (65\%) presented with mild course of the disease (PCDAl: 7.5-27.5). Baseline FCP > $200 \mathrm{mg} / \mathrm{kg}$ was reported in $90 \%$ of patients. Before EEN commencement, undernutrition was observed in $30 \%$ of children, which was established by BMI below the third percentile according to Polish charts. In $25 \%$ of patients, a growth deficit was observed (defined as growth below third percentile) before nutritional therapy introduction (Table I).

The mean decrease in PCDAI score following EEN commencement was statistically significant (from 25.6 \pm 12 to $5.4 \pm 10, p<0.05$ ) (Figure 1 ).

Full remission (defined as PCDAI $<10$ ) was achieved in $65 \%$ of patients, clinical response in another $30 \%$ of them, and $5 \%$ of children did not respond to the treatment.

At week 10 (4 weeks after the end of EEN) a statistically significant decrease in FCP level was observed. Mean values of $3380 \pm 7746$ at baseline declined to $1046.6 \pm 1219$ at week 10, $p<0.05$ (Figure 2). However, complete mucosal healing was stated only in $25 \%$ of patients.

Table II presents values (mean and range) of inflammatory markers (PCDAI and FCP) at baseline and week 10.

All patients, apart from 1 who was fed with a nasogastric tube, accepted oral intake of industrial formula. EEN was generally well tolerated. Initially in $20 \%$ of patients the symptoms of industrial diet intolerance were observed, but they receded within the first days of the therapy. The intolerance was manifested as stomach ache in 2 patients and nausea in 2 other cases.

The recommended daily intake of the formula was achieved in $95 \%$ of children. Only one child was unable

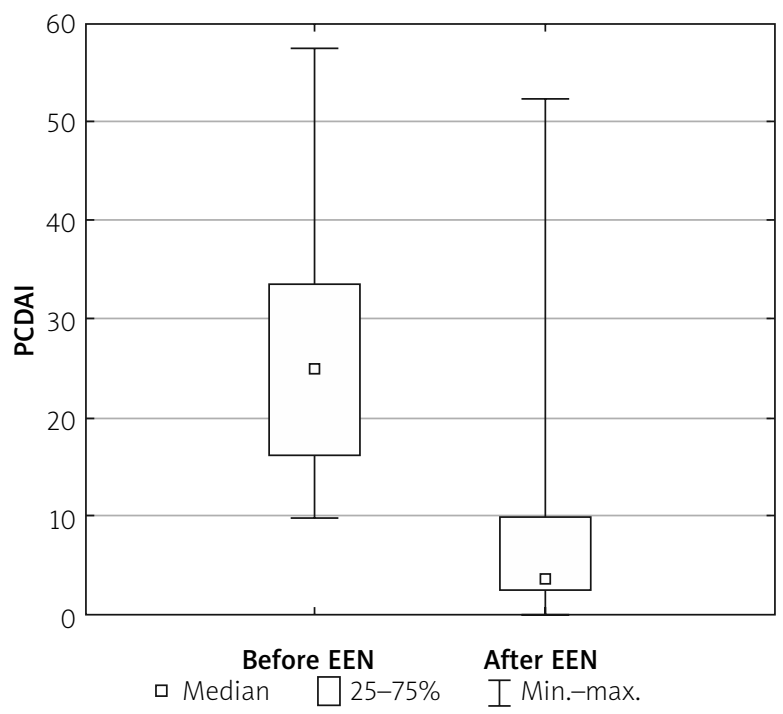

Figure 1. Decrease in PCDAI score following nutritional therapy with EEN 


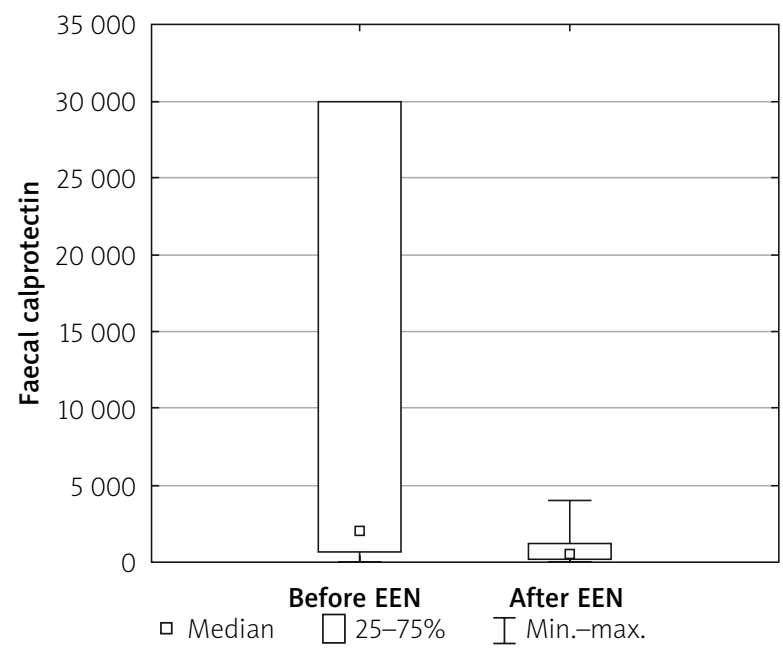

Figure 2. Decline in faecal calprotectin levels after EEN commencement

to intake the prescribed amount of the diet due intolerance.

Improvement in BMI status was reported at the end of the treatment in all patients. The mean increase in BMI score was 0.91, and it was greater in the malnourished group compared to patients with normal nutritional status ( 1.19 vs. 0.62, respectively). After the treatment two-thirds of children with malnutrition had their $\mathrm{BMI}$ score within the normal range. The overall increase in body height was obtained in $55 \%$ of children and in $80 \%$ of those with initial growth failure. The mean growth gain was $1 \mathrm{~cm}$, and it was greater in the group with initial growth deficit relative to patients with normal height values for age at baseline $(1.5 \mathrm{~cm}$ vs. $0.8 \mathrm{~cm}$, respectively) (Table III).

\section{Discussion}

According to the current guidelines of the European Crohn's and Colitis Organization (ECCO), EEN is recommended as first-line therapy to induce remission in children with active luminal CD [17]. Partial EN should not be used for induction of remission. The duration of EEN as induction therapy is usually 6-8 weeks. In our study all patients received EEN for 6 weeks, as recommended.

Because nutritional treatment promotes $\mathrm{MH}$, restores bone mineral density, and improves growth, it should be preferred over corticosteroids for all children with inflammatory intestinal luminal disease, including colonic involvement $[17,25]$. However, in clinical practice, due to the necessity for patient's high motivation, especially when nasogastric tube placement is needed, in some cases EEN is more difficult to commence than pharmacotherapy.
Table II. Markers of disease activity at baseline and after nutritional treatment with EEN $(n=20)$

\begin{tabular}{lccc} 
Parameter & Baseline & Week 10 & P-value \\
\hline PCDAl (mean) & $25.6 \pm 12$ & $5.4 \pm 10$ & \\
\hline PCDAl (range) & $10-57.5$ & $0-15$ & 0.000132 \\
\hline Faecal calprotectin & $3380 \pm 7746$ & 1046.6 & \\
{$[\mathrm{mg} / \mathrm{kg}$ ] (mean) } & & \pm 1219.0 & \\
\hline Faecal calprotectin & $28->30,000$ & $25.0-4006.0$ & 0.000892 \\
{$[\mathrm{mg} / \mathrm{kg}$ ] (range) } & & & \\
PCDAl - paediatric Crohn's disease activity index. &
\end{tabular}

Table III. Patients' anthropometric characteristics (body mass, height, and BMI) at baseline and after EEN commencement $(n=20)$

\begin{tabular}{lcc} 
Parameter & Baseline & Week 10 \\
\hline Body mass $[\mathrm{kg}]$ (mean) & $43.0 \pm 12.4$ & $45.4 \pm 12.0$ \\
\hline Body mass $[\mathrm{kg}]$ (range) & $25-72$ & $27.5-72$ \\
\hline Body height $[\mathrm{cm}]$ (mean) & $156.7 \pm 13.5$ & $157.8 \pm 13.3$ \\
\hline Body height $[\mathrm{cm}]$ (range) & $133-176$ & $133-176$ \\
\hline BMI [kg/m²] (mean) & $14.97 \pm 10.1$ & $15.5 \pm 9.5$ \\
\hline BMI [kg/m²] (range) & $12.6-25$ & $13-25.4$
\end{tabular}

At present, no placebo-controlled randomized controlled trial (RCT) of EEN with exclusive liquid formula feeds has been conducted in children with CD, but there have been several RCTs comparing EEN to standard treatment.

Three meta-analyses have demonstrated that the overall combined remission rate for EEN in paediatric $C D$ is $73 \%$ (relative risk $(R R)=0.95,95 \%$ confidence interval (Cl): $0.67-1.34$ and $\mathrm{RR}=0.97,95 \% \mathrm{Cl}$ : 0.7-1.4) $[4,5,26]$. The results from our study are comparable: $65 \%$ of patients entered full clinical remission following nutritional therapy with EEN.

According to published data, $\mathrm{MH}$ rates in children treated with EEN have ranged from $19 \%$ to $75 \%$ [27-30]. However, differences in the definition of $\mathrm{MH}$ in these studies limit the ability to summarize the results. The only RCT assessing $\mathrm{MH}$ as an outcome of EEN with Modulen IBD compared with corticosteroids demonstrated a clear superiority after 8 weeks of EEN, with rates of $89 \%$ vs. $17 \%$, respectively [8] In that study $\mathrm{MH}$ was assessed in endoscopy while in our study FCP was used to define $\mathrm{MH}$ because this biomarker seems to correlate best with endoscopic examination and is considered as good marker of $\mathrm{MH}$ [31]. At week 10 we observed a statistically significant decrease in FCP levels, which may suggest that exclusive enteral nutrition promotes healing of intestinal mucosa as well. How- 
ever, only $25 \%$ of patients achieved complete mucosa healing defined as FCP below $200 \mathrm{mg} / \mathrm{kg}$. A likely explanation for this unsatisfactory result is that the control measurement of calprotectin was done 4 weeks after the end of EEN and the start of the regular diet. Recently Levine et al. showed that after 6 weeks of EEN with "Modulen IBD" the FCP levels dropped significantly, but they increased again at week 12 (6 weeks after the reintroduction of standard food) [24]. Maybe if we performed the measurement of FCP at week 6, immediately after the end of the EEN, the results would have been better. Moreover, in the study by Levine et al., after 6 weeks of EEN, only $15 \%$ of children achieved complete $\mathrm{MH}$ defined in the same way as in our study, i.e. FCP below $200 \mathrm{mg} / \mathrm{kg}$. The authors explained the poor results by the very high calprotectin levels at enrolment. The median baseline FCP level in their patients was comparable to ours, i.e. 2647 vs. $3380 \mu \mathrm{g} / \mathrm{kg}$, respectively. The high FCP levels at baseline (meaning huge mucosal damage) is another potential explanation of the low rate of patients with complete $\mathrm{MH}$ after 6 weeks of EEN in our group.

Hartman et al. performed a retrospective study including the charts of 28 patients, to summarize the experience of several paediatric gastroenterology units in Israel using "Modulen IBD” supplementation in children and adolescents with CD [7]. They observed that a diet with "Modulen IBD" was associated with a statistically significant decrease in PCDAI, from 34.3 to 15.7 ( $p<$ 0.0001 ), which is consistent with our findings. Moreover, similarly to our study, the children supplemented with "Modulen IBD" also showed improvement in BMI, suggesting an additional advantage of nutritional therapy in CD patients.

A Spanish study assessing the efficacy of 6-8-week EEN as a primary therapy for children with CD (14 patients included in the study) and its impact on $\mathrm{MH}$ (defined by FCP, as in our study) demonstrated that after 8 weeks of EEN, $85 \%$ of patients were in clinical remission and FCP levels declined significantly without reaching normal levels [13]. These results confirm that the duration of EEN as induction therapy should be 6-8 weeks, and it takes more time to promote $\mathrm{MH}$; FCP levels, although significantly reduced, were still above normal levels. In our study fewer (65\%) patients were in clinical remission after EEN commencement, which may be due to shorter duration of nutritional treatment (6 vs. 8 weeks), which also suggests that the longer EEN is provided, the better the outcomes. Moreover, Levine et al. showed the remission rate, defined as in our study as PCDAI < 10 points, was lower at week 6 than at week 12 ( 6 weeks after the end of EEN and the start of regular diet) [24]. In our group the PCDAI was assessed 4 weeks after the end of EEN, which could be why the remission rate was lower than in the Spanish study where PCDAI was evaluated immediately after the end of EEN [13].

Although most published studies have proven the efficacy of EEN in induction of remission in paediatric CD patients, there is still an unresolved issue. Comparing our results with others we can conclude that the 6-week course of EEN led to a decline in FCP level but was too short to induce complete mucosal healing, especially in patients with high baseline FCP results. The second point is to pay attention to the lack of a strategy to reintroduce normal food after the EEN. In our patients the remission rate was lower than in studies where the assessment was done immediately after the end of nutritional therapy $[13,24]$. This suggests that after EEN a special diet is needed to maintain remission. There are promising results from a study by Levine et al. - they showed that partial enteral nutrition plus a special diet (Crohn's Disease Exclusion Diet) has the same effectiveness as EEN in induction of remission in children with Crohn's disease. This new idea of nutritional treatment is easier to apply in patients, and it defines the next stages of re-introduction of as normal diet [24].

Nonetheless, more properly designed studies are needed to establish the optimal method of nutritional therapy in paediatric CD patients.

\section{Conclusions}

In our study including 20 children with active CD a 6-week course of EEN was an effective and well tolerated method of treatment. Nutritional therapy induced full clinical remission and led to a decline in FCP level (as marker of $\mathrm{MH}$ ) in the majority of patients, but it was not sufficiently effective to induce complete mucosal healing. Another important benefit from that treatment was the improvement of the children's nutritional status and growth velocity.

\section{Conflict of interest}

The authors declare no conflict of interest.

\section{References}

1. Veauthier B, Hornecker JR. Crohn's disease: diagnosis and management. Am Fam Physician 2018; 98: 661-9.

2. Vasseur F, Gower-Rousseau C, Vernier-Massouille G, et al. Nutritional status and growth in Pediatric Crohn's Disease: a population-based study. Am J Gastroenterol 2010; 105: 1893-900.

3. Sanderson IR. Growth problems in children with IBD. Rev Gastroenterol Hepatol 2014; 11: 601-10.

4. Heuschkel RB, Menache CC, Megerian JT, Baird AE. Enteral nutrition and corticosteroids in the treatment of acute Crohn's disease in children. J Pediatr Gastroenterol Nutr 2000; 31: 8-15. 
5. Dziechciarz P, Horvarth A, Shamir R, Szajewska H. Meta-analysis: enteral nutrition in active Crohn's disease in children. Aliment Pharmacol Ther 2007; 26: 795-806.

6. Day AS, Whitten KE, Sidler MI, Lemberg DA. Systematic review: nutritional therapy in Paediatric Crohn's Disease. Aliment Pharmacol Ther 2007; 27: 293-307.

7. Swaminath A, Feathers A, Ananthakrishnan AN, et al. Sys tematic review with meta-analysis: enteral nutrition therapy for the induction of remission in paediatric Crohn's disease. Aliment Pharmacol Ther 2017; 46: 645-56.

8. Pigneur B, Lepage P, Mondot S, et al. Mucosal healing and bacterial composition in response to enteral nutrition vs steroid-based induction therapy-a randomised prospective clinical trial in children with Crohn's disease. J Crohns Colitis 2019 13: 846-55.

9. Freeman HJ. Natural history of Crohn's disease: com-parison between childhood- and adult-onset disease. Inflamm Bowel Dis 2010; 16: 953-61.

10. Van Limbergen J, Russell RK, Drummond HE, et al. Definition of phenotypic characteristics of childhood-onset inflammatory bowel disease. Gastroenterology 2008; 135: 1114-22.

11. Vernier-Massouille G, Balde $M$, Salleron J, et al. Natural history of pediatric Crohn's disease: a population-based cohort study. Gastroenterology 2008; 135: 1106-13.

12. Sanderson IR, Boulton P, Menzies I, Walker-Smith JA. Improvement of abnormal lactulose/rhamnose permeability in active Crohn's disease of the small bowel by an elemental diet. Gut 1987; 28: 1073-6.

13. Leach ST, Mitchell HM, Eng WR, et al. Sustained modu-lation of intestinal bacteria by exclusive enteral nutrition used to treat children with Crohn's disease. Aliment Pharmacol Ther 2008; 28: 724-33.

14. Lionetti P, Callegari ML, Ferrari S, et al. Enteral nutrition and microflora in pediatric Crohn's disease. JPEN J Parenter Enteral Nutr 2005; 29: S173-5.

15. Hartman C, Berkowitz D, Weiss B, et al. Nutritional supplementation with polymeric diet enriched with transforming growth factor-beta 2 for children with Crohn's disease. Isr Med Assoc J 2008; 10: 503-7.

16. Fell JM. Control of systemic and local inflammation with transforming growth factor beta containing formulas. J Parenter Enteral Nutr 2005; 29 (4 Suppl): S126-8.

17. Ruemmele FM, Veres G, Kolho KL, et al. Consensus Guidelines of ECCO/ESPGHAN on the Medical Management of Pediatric Crohn's Disease. Crohns Colitis 2014; 8: 1179-207.

18. Rubio A, Pigneur B, Garnier-Lengline $\mathrm{H}$, et al. The efficacy of exclusive nutritional therapy in paediatric Crohn's disease, comparing fractionated oral vs. continuous enteral feeding. Aliment Pharmacol Ther 2011; 33: 1332-9.

19. Morikawa M, Derynck R, Miyazono K. TGF- $\beta$ and the TGF- $\beta$ family: context-dependent roles in cell and tissue physiology Harb Perspect Biol 2016; 8: a021873.

20. Yamamoto T, Shimoyama T, Kuriyama M. Dietary and enteral interventions for Crohn's disease. Opin Biotechnol 2017; 44 69-73.

21. Forbes A, Escher J, Hébuterne X, et al. ESPEN guideline: clinical nutrition in inflammatory bowel disease. Clin Nutr 2017; 36: 321-47.
22. Navas López VM, Blasco Alonso J, Sierra Salinas C, et al. Efficacy of exclusive enteral feeding as primary therapy for paediatric Crohn's disease. An Pediatr 2008; 69: 506-14.

23. Lionetti $P$, Callegari ML, Ferrari S, et al. Enteral nutrition and microflora in pediatric Crohn's disease. J Parenter Enteral Nutr 2005; 29 (4 Suppl): S173-5.

24. Levine A, Wine E, Assa A, et al. Crohn's disease exclusion diet plus enteral nutrition induces sustained remission in a randomized controlled trial. Gastroenterology 2019; 157: 440-50.

25. Limketkai BN, Wolf A, Parian AM. Nutritional interventions in the patient with inflammatory bowel disease. Gastroenterol Clin North Am 2018; 47: 155-77.

26. Zachos M, Tondeur M, Griffiths AM. Enteral nutritional therapy for induction of remission in Crohn's disease. Cochrane Database Syst Rev 2007; 1: Cd000542.

27. Bannerjee K, Camacho-Hubner C, Babinska K, et al. Anti-inflammatory and growth-stimulating effects precede nutritional restitution during enteral feeding in Crohn disease. J Pediatr Gastroenterol Nutr 2004; 38: 270-5.

28. Afzal NA, Van Der Zaag-Loonen HJ, Arnaud-Battandier F, et al. Improvement in quality of life of children with acute Crohn's disease does not parallel mucosal healing after treatment with exclusive enteral nutrition. Aliment Pharmacol Ther 2004; 20: 167-72

29. Fell JM, Paintin M, Arnaud-Battandier F, et al. Mucosal healing and a fall in mucosal pro-inflammatory cytokine mRNA induced by a specific oral polymeric diet in paediatric Crohn's disease. Aliment Pharmacol Ther 2000; 14: 281-9.

30. Berni Canani R, Terrin G, Borrelli O, et al. Short- and long-term therapeutic efficacy of nutritional therapy and corticosteroids in paediatric Crohn's disease. Dig Liver Dis 2006; 38: 381-7.

31. Sipponen T, Kolho KL. Fecal calprotectin in diagnosis and clinical assessment of inflammatory bowel disease. Scand J Gastroenterol 2015; 50: 74-80.

Received: 30.11 .2020

Accepted: 10.03.2021 